\title{
Fluid adsorption in linear pores: a molecular simulation study of the influence of heterogeneities on the hysteresis
} loop and the distribution of metastable states.

\author{
Joël Puibasset \\ Centre de Recherche sur la Matière Divisée, CNRS-Université d'Orléans, 1b rue de la \\ Férollerie, 45071 cedex 02, France
}

\begin{abstract}
:
Porous materials are known to adsorb fluid, and can be characterized by measurement of fluid adsorption isotherms. Many nanoporous materials exhibit linear pores, like MCM-41, porous silicon or alumina, etc. In such systems, data adsorption analysis is considered to be straightforward within the approximation of independent domains. This paper, which reviews previous molecular simulation works, aims at showing that the presence of heterogeneities within the pores actually invalidates this hypothesis, with consequences for porosity characterization. To enlighten the effects, starting from perfect cylinders, the number of heterogeneities is progressively increased, up to large numbers, for which specific simulation tools are used to take into account the interdependence between the domains. The adsorption/desorption isotherms are calculated, and correlated to the appearance of an exponentially large number of metastable states.
\end{abstract}




\section{INTRODUCTION}

Essentially all surfaces adsorb fluids, due to molecular interactions [1-4]. These interactions may be weak (van der Waals or electrostatic) or much stronger. For strong interactions, chemical bonds may be broken in the adsorbed molecules and/or established between the adsorbent and the adsorbate: this is chemisorption. On the other hand, for weak interactions, the adsorption process occurs reversibly without breaking any molecule: this is physisorption. The paper will focus on this situation. Despite weak, the fluid/substrate interactions induce observable modifications of the fluid properties in the vicinity of the surface. Stronger effects may be induced when fluids are confined in porous materials, because the local curvature associated to porosity enhances fluid/substrate interactions. As an example, one observes that porous materials condense gases below their saturating point. The measured adsorption isotherm (giving the amount of fluid adsorbed versus fluid pressure) thus provides a powerful experimental tool for porous materials characterization $[3,4]$. In practice, it is not a straightforward task to assess the pore geometry from the isotherms. For instance, the local surface chemistry may enhance or disfavor adsorption: a famous example is given by the hydroxyl groups $\mathrm{OH}$ which influence the hydrophilic property of silica [5-17]. Another important case for environmental purpose regards the adsorption of condensable gases like carbon dioxide or methane on porous carbon-based materials. See for example the recent work by Wilcox et al.[18-22] on the influence of surface chemistry and pore network on adsorption.

An important feature of such adsorption isotherms, which increases the difficulty of their analysis, is their possible irreversibility: desorption do not coincide with adsorption (existence 
of a hysteresis, to be discussed later). Furthermore, the size of the reservoir used to perform adsorption experiments has to be chosen carefully, i.e. large enough to avoid spurious effects, a condition which is not so frequently fulfilled since "large" should be understood in terms of number of molecules between the system and the reservoir and not in terms of volumes [2325]. Data analyses also have to take into account corrections arising from an accurate reconsideration of physics of adsorption [26-28].

Focusing on heterogeneous surfaces, we show how surface chemistry and geometry influence the adsorption properties of a condensable fluid and hence its liquid/vapor coexistence diagram. In most cases, molecular simulation [29-31], taking into account the fluid/substrate interactions at atomistic scale, has proven to be a powerful tool to understand adsorbed fluid properties in the vicinity of a surface. In particular for studying the effect of surface chemistry or corrugation $[32,33]$, local curvature, pore morphology [34, 35], or any other nanometerscale property of the surface [36].

We show that, increasing the surface heterogeneities has drastic effects on coexistence diagram. Not only a shift of the critical point (i.e. quantitative changes), but also the appearance of many metastable states that introduce qualitative changes [37-41]. A multiscale approach has been used to explore this issue, showing the importance of the interdependence between the various heterogeneities for the adsorption and desorption mechanisms, the observed hysteresis, and the distribution of the underlying metastable states $[42,43]$. Such approach, including both an atomistic and a large-scale description (i.e. from interatomic forces to large scale domain network) is expected to offer a powerful tool for experimental data analysis. 


\section{MOLECULAR MODELS}

The main objective of this simulation work is to describe trends in adsorption due to the presence of heterogeneities, ranging from one single heterogeneity to a large number. The model needs to be simple to catch the main features of adsorption, avoiding unnecessary (and possibly misleading) details. This is why the interactions are chosen to be extremely simple, with however the realistic orders of magnitude. Similarly, the heterogeneities will be generated in the simplest possible way, as described in the following sections.

All (fluid-fluid and fluid-wall) interactions are described by the (12-6) Lennard-Jones interatomic potential cut at $2.5 \sigma$ (interactions are not calculated beyond that distance) and shifted (the potential reaches zero at cutoff). The adsorbate is chosen to be argon, a simple fluid commonly used for porous materials characterization. The parameters for argon are $\varepsilon_{\mathrm{Ar}-}$ ${ }_{\mathrm{Ar}} / k=119.8 \mathrm{~K}$ where $k$ is Boltzmann's constant, and $\sigma_{\mathrm{Ar}-\mathrm{Ar}}=0.3405 \mathrm{~nm}$. The fluid-fluid parameters provide natural units to measure the physical quantities introduced in this paper (the unit for distances is $\sigma_{\mathrm{Ar}-\mathrm{Ar}}$; for energies it is $\varepsilon_{\mathrm{Ar}-\mathrm{Ar}}$; for temperatures it is $\varepsilon_{\mathrm{Ar}-\mathrm{Ar}} / k$; for pressures it is $\varepsilon_{\mathrm{Ar}-\mathrm{Ar}} / \sigma_{\mathrm{Ar}-\mathrm{Ar}}{ }^{3}$; etc). The reduced quantities will be denoted by an asterisk. The parameters for argon/wall interactions are $\varepsilon_{\mathrm{Ar}-\mathrm{W}} / k=100 \mathrm{~K}$ and $\sigma_{\mathrm{Ar}-\mathrm{W}}=0.333 \mathrm{~nm}$. These parameters are typical for argon-oxygen interactions, corresponding to the main contribution for argon/silica interaction. The adsorbent (pore) is chosen to be a cylinder of $3 \mathrm{~nm}$ in diameter. The smooth wall approximation may be used: it consists in averaging the fluid-wall interaction over the atomic roughness (the potential is purely radial in this case). Differences in adsorption isotherms are negligible (small differences at low pressure only), but it allows to save computing time. 
We focus on simple fluid adsorption in the frequently encountered silica-based nanoporous materials like MCM-41 or oxidized porous silica. These materials exhibit parallel and noninterconnected tubular pores with a high aspect ratio (few nanometers in diameter and few microns of length) [44-46]. Such materials are of greatest fundamental interest since they exhibit unexpected hysteresis shapes [47-49]. Due to fabrication procedure, one expects heterogeneities at nanometer scale along the pore axis [50-54]. These heterogeneities result from variations of local surface chemistry (the density of surface hydroxyls groups may vary for instance) or from variations in geometric characteristics (constrictions in pores). These heterogeneities essentially result in a modulation of the affinity of the adsorbed fluid (argon in our case) with the wall. We have in particular shown that pore size variations actually predominantly result in variations of fluid/wall interactions, with moderate "purely geometric" effects [55-58]. As a simplification, the corresponding heterogeneities are characterized by a simple parameter which correlates directly to the local intensity of the fluid/wall interaction. More precisely, this parameter is an extra factor applied to the fluid/wall interaction. Values less than 1 (down to 0.7 in our model) produce domains with lower affinity for the fluid. Values larger than 1 (up to 1.3 in our model) produce domains with higher affinity for the fluid. This parameter is varied along the pore axis to produce heterogeneities. Several cases will be considered: no heterogeneity, one single heterogeneity, two heterogeneities with different amplitudes, and a large number of heterogeneities with random amplitude taken from a given (Gaussian) distribution. The first case corresponds to a perfectly cylindrical pore, chosen to be of diameter $3 \mathrm{~nm}$ and length $L=4.086 \mathrm{~nm}=12 \sigma_{\mathrm{Ar}-\mathrm{Ar}}$ (see Fig. 1-a). For the second case, the heterogeneity is produced by modulating the fluid-wall potential of the perfectly cylindrical pore by the extra factor $\left\{1+0.25 \cos \left(\frac{2 \pi z}{L}\right)\right\}$, where $L$ is the pore length and $\mathrm{z}$ is the coordinate along the pore axis. This corresponds to a sinusoidal 
modulation along the pore axis of 25 percent maximum. The pore is thus a chain (due to
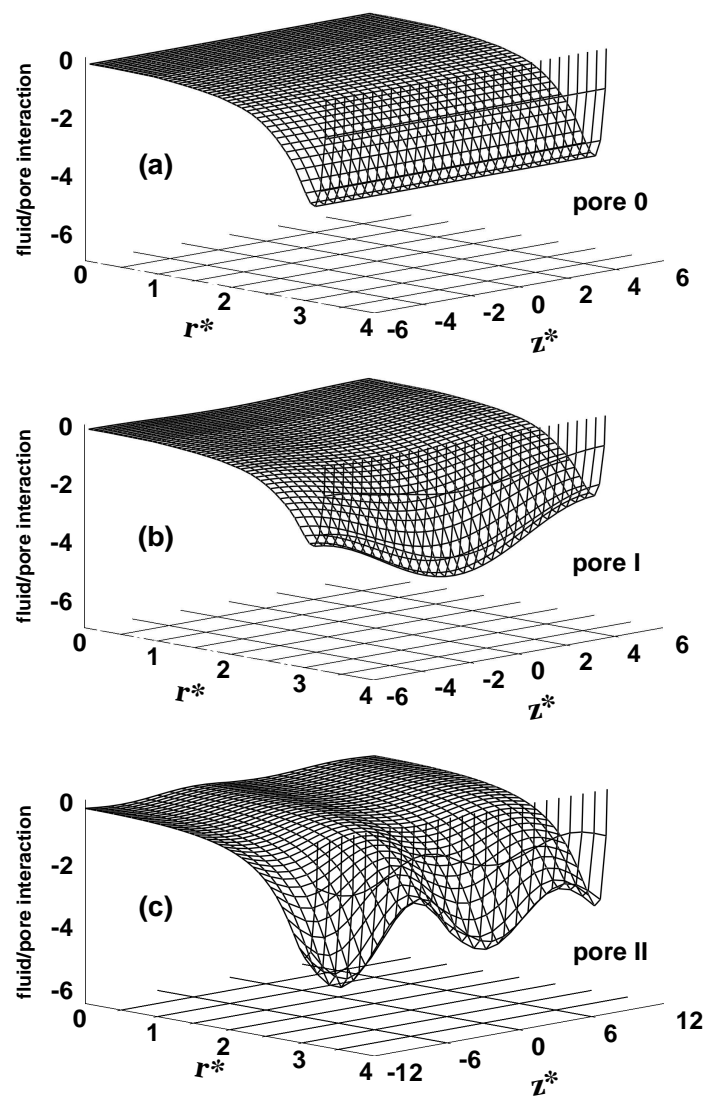

(d) pore III: example with 140 domains
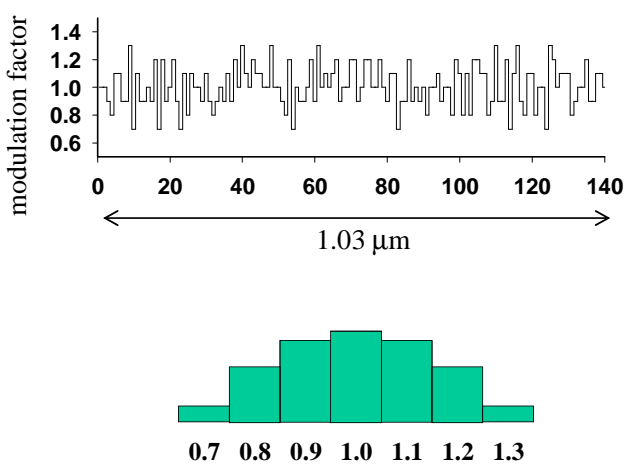

Figure 1: Presentation of the four pore models. a) pore 0: fluid/wall potential in reduced units for the perfectly cylindrical pore (length $12 \sigma_{\mathrm{Ar}-\mathrm{Ar}}$ ); $r^{*}$ is the reduced radial coordinate and $z^{*}$ is the reduced coordinate along the pore axis; b) pore I: fluid/wall potential in reduced units for the pore with one single sinusoidal heterogeneity deduced from pore 0 by sinusoidal modulation along $z^{*}$ (length $12 \sigma_{\mathrm{Ar}-\mathrm{Ar}}$ ); c) pore II: fluid/wall potential in reduced units for the pore with two sinusoidal heterogeneities of different strength, giving rise to four domains with different fluid/wall affinities (length: $24 \sigma_{\mathrm{Ar}-\mathrm{Ar}}$ ); d) pore III (many heterogeneities): modulation factor along the domains (140 in this example, corresponding to a 1 micrometer long pore); the values are taken from the Gaussian distribution shown below. 


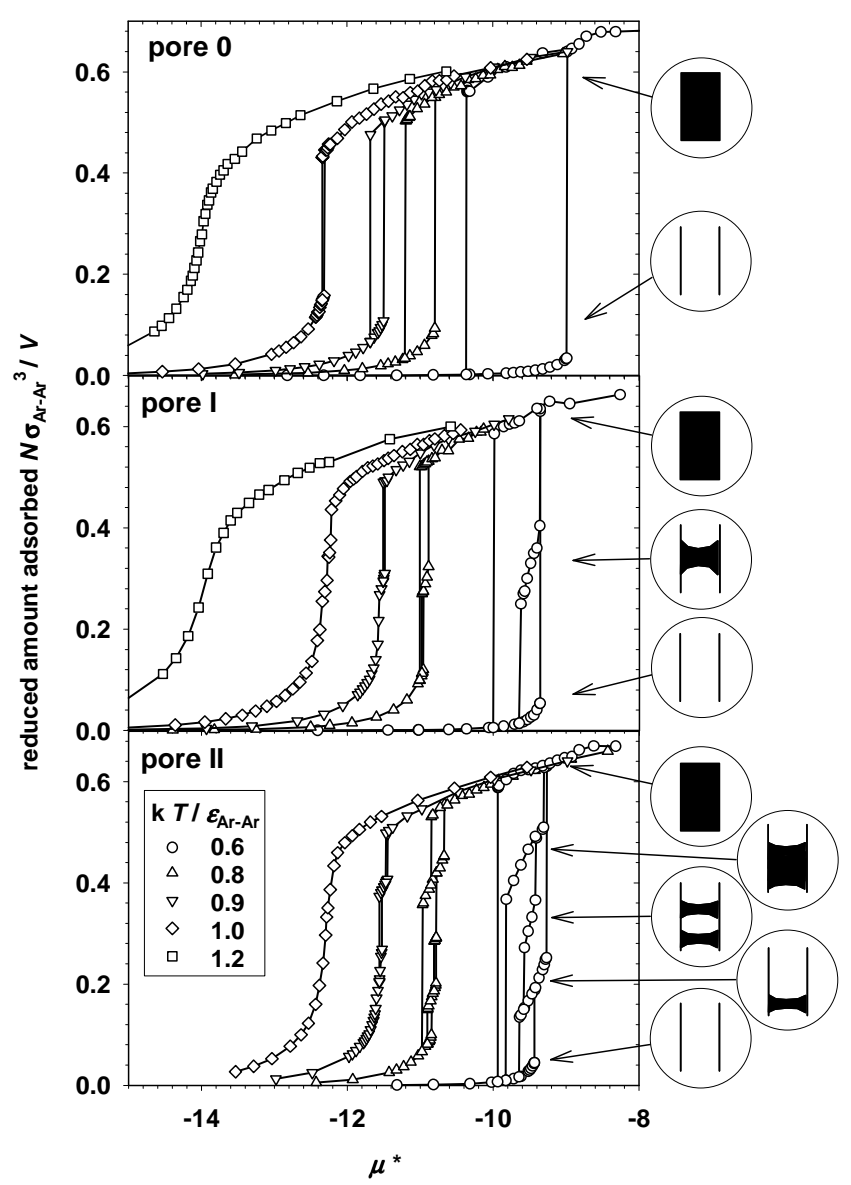

Figure 2: Adsorption/desorption isotherms for argon confined in the three systems with few heterogeneities obtained by Grand Canonical Monte Carlo simulation (symbols). The reduced temperatures are given in the inset. Lines are guide to the eye. The vertical lines materialize the system jumps when the metastability limits are exceeded. The large circles contain schematic representation of the fluid distribution in the pores (black: high density liquidlike fluid; white: low density gaslike fluid).

periodic boundary conditions) of more or less attractive regions (see Fig. 1-b). In the third case, the modulation factor is $\left\{1+a(z) \sin \left(\frac{4 \pi z}{L}\right)\right\}$ where $\mathrm{a}=0.3$ for $\mathrm{z}<0$ and $\mathrm{a}=0.2$ for $\mathrm{z}>0$, and $L=8.172 \mathrm{~nm}=24 \sigma_{\mathrm{Ar}-\mathrm{Ar}}$ is the pore length. This produces four regions per period in the pore with high, medium high, medium low and low fluid/wall affinities (see Fig. 1-c). In the last case, many modulations are introduced in the pore. For simplicity a step function has been used: the modulation factor is constant within each domain (of length $7.36 \mathrm{~nm}=21 \sigma_{\mathrm{Ar}-\mathrm{Ar}}$ ), 
and takes uncorrelated values between two adjacent domains. Its value is supposed to follow a Gaussian distribution (see Fig 1-d).

The simulations are performed using the grand canonical Monte Carlo (GCMC) algorithm [29-31]. The chemical potential $\mu$ of argon is imposed and related to the gas pressure in the reservoir using the ideal gas relation since it is a good approximation at the temperature at which the simulations are performed (for instance $T^{*}=T k / \varepsilon_{\mathrm{Ar}-\mathrm{Ar}}=0.6$ corresponds approximately to $70 \mathrm{~K}$ ). The adsorption/desorption isotherms are given as a function of the reduced chemical potential $\mu^{*}=\mu / \varepsilon_{\mathrm{Ar}-\mathrm{Ar}}$. Long simulations are performed in order to reduce the uncertainties down to $7 \%\left(10^{8}\right.$ Monte Carlo trials per argon where required).

\section{PORES WITH FEW HETEROGENEITIES: SIMULATION RESULTS}

Figure 2 shows the adsorption/desorption isotherms for fluid adsorbed in a cylindrical pore containing 0,1 or 2 sinusoidal heterogeneities. At low temperature (below the hysteresis critical temperature) the isotherms exhibit hysteresis. The points actually group into branches along which the adsorption/desorption is reversible. These branches correspond to deep local minima in the system's free energy landscape (stable or metastable states), separated from other minima by energetic barriers larger than thermal fluctuations. The system evolves reversibly upon adsorption or desorption along the branches, at least within the $\mu$ limits beyond which the energetic barriers become small enough to be overcome by thermal fluctuations (i.e. the stability limit of the metastable state has been reached and the system jumps into a deeper adjacent local minimum). The vertical lines in Fig 2 indicate the new local minima reached by the system. An important observation is that the number of 
metastable states is higher at low temperature, and increases significantly with the number of heterogeneities.

Let us now give a molecular picture for these local minima. The lowest density branch corresponds to gaslike fluid filling the pores with molecules adsorbed at the walls. The highest density branch corresponds to pore completely filled by liquidlike fluid. These states, observable in the three systems, are reminiscent of the bulk fluid. New metastable states are stabilized by the chemical corrugation. Visual inspection of molecular configurations in the pore made of a single heterogeneity shows that the local minimum of intermediate density corresponds to a liquidlike meniscus located in the strongly attractive domain of the pore. This local minimum is the analog in heterogeneous cylindrical pores of the bridgelike phase observed in corrugated slit pores [32]. See schematic representations in Fig. 2.

In the pore with two sinusoidal heterogeneities, five local minima are observed, which correspond respectively to: a gaslike fluid adsorbed in the pore; one liquidlike meniscus in the most attractive domain of the pore; two liquidlike menisci in the two attractive regions of the pore; one single large meniscus bridging the two attractive domains; a liquidlike fluid completely filling the pore (Fig. 2). This system shows very interesting features. As an example, for a reduced temperature of 0.8 , starting from the empty system and gradually increasing the chemical potential results in continuous filling of the system up to $\mu=-10.84$ where the system jumps into the second local minimum. Increasing further the chemical potential makes the system jump successively into the third, fourth and finally the fifth local minimum. Upon desorption, the system does not visit the local minima in the reverse order. After the first step, which consists in the formation of a bubble in the less attractive domain (i.e. the system jumps from the fifth into the fourth local minimum), further decrease in 
chemical potential makes the system jump directly into the first local minimum (gaslike branch) without visiting the third and second minima. This is an important point which shows that it is possible to miss metastable states when performing simple adsorption or desorption cycles [59].

This simple model shows that the hysteresis loop is related to a complex free energy landscape, and may present a dissymmetry in adsorption and desorption. It is also shown that the main adsorption and desorption isotherms are made of several branches with small discontinuities. Furthermore, a simple picture of adsorption/desorption subloops (scanning curves) arises, as irreversible paths between the various local minima.

\section{PORE WITH A LARGE NUMBER OF HETEROGENEITIES: MULTISCALE} APPROACH

Let us now consider pores with a large number of domains. Such systems are too large to be treated as a whole with usual molecular simulation tools. Let us introduce the multi-scale molecular simulation approach, which enables to study pores with a large number of heterogeneities. As introduced by Everett [60-62] a porous material can be seen as a collection of domains corresponding to elemental subdivision of the porous network (elemental cavities). Most of the time, the domains are considered to be independent to simplify the calculations (see Fig. 3). The global thermodynamic properties are thus obtained as an average over the domain distribution. Molecular simulation is able to treat separately each domain of nanometric scale, and thus provides a powerful analysis of fluid adsorption after averaging over the appropriate distribution of domains. This approach leads to an extremely 
accurate description of the low pressure region of the adsorption isotherm, where the hypothesis of independence between domains is well justified. But the hysteresis region is not well described by this approach because the disorder associated to the complex porous network plays an important role [43, 63-69].

As a matter of fact, in the hysteresis region, the amount of fluid adsorbed in any domain is not a single valued function of the chemical potential. It also depends on the fluid state in the neighboring domains (this is closely connected to the existence of multiple metastable states). It is possible to approximately rationalize this dependence from a classical thermodynamic analysis of fluid adsorption in pores. However, significant improvement can be achieved if one uses molecular simulation to extract them, with appropriate explicit boundary conditions to take into account the presence of neighboring domains [70, 71].

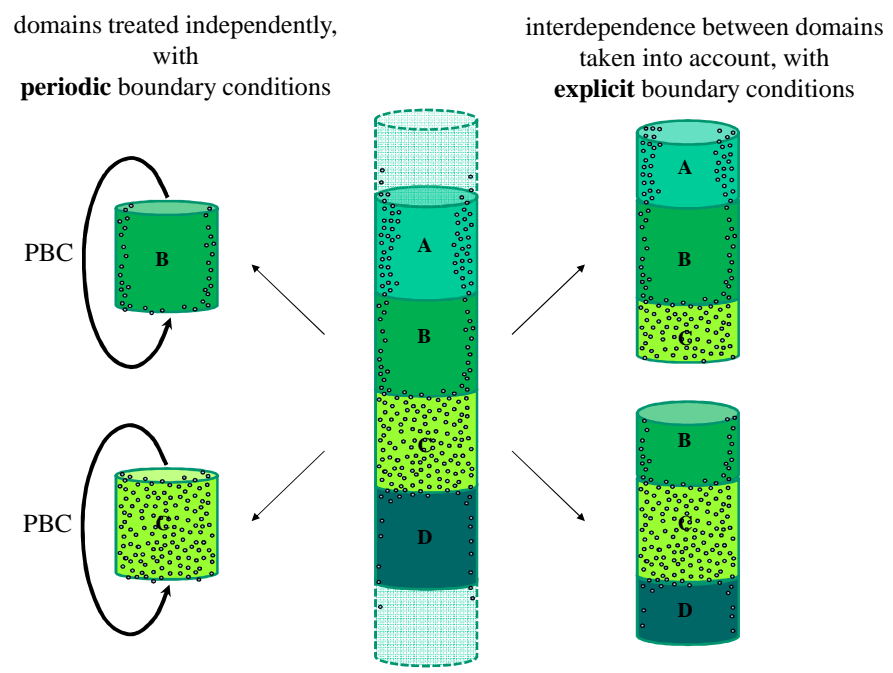

Figure 3: Schematic representation of a heterogeneous pore (in the center; four different domains labeled A to D can be seen). The system may either be treated as if the domains where independent (left, all domains are treated independently with periodic boundary conditions, PBC) or as a whole (right, the interdependence between domains is taken into account by means of explicit boundary conditions, see text). 
This is possible because interactions and correlations are short range: as a consequence, only the (two) nearest neighboring domains need to be considered. Actually, only the portions of the neighboring domains within interaction range need to be considered for the calculations (the remaining part beyond the interaction range has no direct influence on the central domain). Furthermore, the main effect of the neighboring domains does not originate in their detailed physico-chemical properties: the relevant information is essentially the absence or presence of fluid in the neighboring domains, or, more quantitatively, the corresponding density. Therefore, these neighboring domains may be taken as homogeneous cylindrical pores with the same fluid/wall interactions as the central domain to be studied. The simulation box then contains the central domain (length: $7.36 \mathrm{~nm}=21.6 \sigma_{\mathrm{Ar}-\mathrm{Ar}}$ ) and two adjacent portions of domains (taken to be $3 \sigma_{\mathrm{Ar}-\mathrm{Ar}}$ long) which play the role of boundaries; extra portions are added, where the fluid state (gaslike or liquidlike) is imposed during the calculations (see Fig. 4).

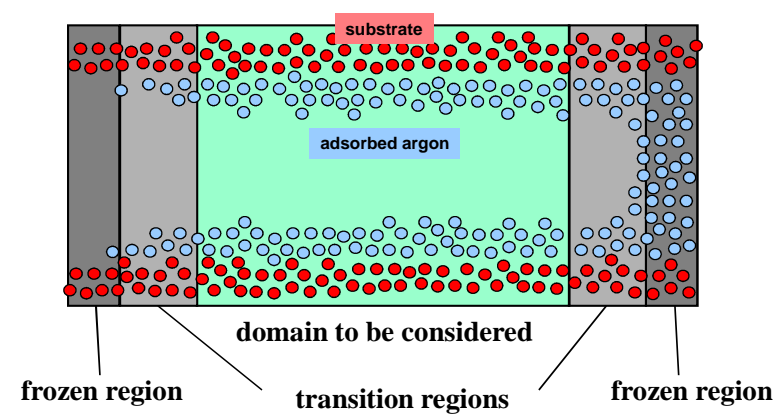

Figure 4: Schematic representation of the simulation box with explicit boundary conditions: the domain to be considered is in the center (green zone); the explicit boundaries are made of frozen regions where the fluid state (gaslike or liquidlike) is imposed and transition regions where the fluid relaxes between its imposed state in the frozen region and its actual state in the domain. Blue circles: adsorbed argon; red circles: substrate species. 
Note that it is not yet known if the neighboring domains are actually filled or empty: we just perform pre-calculations considering all possible hypotheses. In other words, these precalculations aim at determining how the considered domain behaves as a function of the neighboring domains. Essentially, the central domain is bistable (gaslike or liquidlike), and the imposed boundaries forces the system to switch to one state, depending on the chosen boundaries. It's only when all pre-calculations are performed that a real adsorption in the whole pore can be performed. The initial condition being the empty pore, we know that all domains have empty neighbors. The chemical potential is then slowly increased and the precalculated behavior of each domain is used to determine the filling status of each domain, taking into account the status of the neighboring domains. The total amount of fluid adsorbed versus $\mu$ is then deduced (adsorption isotherm). A similar procedure is used for desorption.

\section{PORE WITH A LARGE NUMBER OF HETEROGENEITIES: SIMULATION}

\section{RESULTS}

As for previous systems with few heterogeneities, the main adsorption and desorption isotherms have been calculated by slowly increasing the chemical potential from the initial empty pore up to saturation, and then decreasing the chemical potential until the pore empties completely. Scanning isotherms are obtained in a similar way. However, as seen previously, not all metastable states can be reached with this procedure [59]. To be sure to determine all metastable states for our system, we proceed as follows. We first generate all possible states, characterized by a set of gaslike and liquidlike status for all domains. The total number of possible states is necessarily less than $2^{N}$ (where $N$ is the number of domains) since all domains have at most two possible states. We then eliminate all states which are not 
compatible with the pre-calculated isotherms. From a physical point of view, these configurations correspond to situations where the fluid state is unstable and has to evolve towards a metastable state (local minimum of the free energy). When a new metastable state is found, its stability limits are easily determined by slowly decreasing and/or increasing the chemical potential. The corresponding branches can be drawn in the usual graph giving the amount adsorbed versus chemical potential.

Figure 5 gives the results for a pore containing 15 domains. In this particular system, a total of $\mathcal{N}=135$ metastable states where found. The lowest and highest branches correspond to the usual gaslike and liquidlike states, i.e. fluid adsorbed only at surface or completely filling the pore respectively. The other (metastable) states correspond to partial filling of the pore (some domains may be filled while others are still essentially empty. They appear as portions of lines corresponding to their metastability range. These lines can be seen as the projection in the $\mu-\rho$ plane of the local minima in the free energy landscape. As expected, the slopes are always positive due to increasing film thickness with $\mu$. These lines are almost straight and essentially parallel, which is a remarkable feature. As a consequence, despite eventually great differences in fluid configuration between two adjacent metastable states, the response of the system to a chemical potential variation is essentially the same in both cases. It is also observed that the main hysteresis loop corresponds to the external envelope of the metastable states. This feature is observed in other systems and seems to be quite general $[72,73]$. 


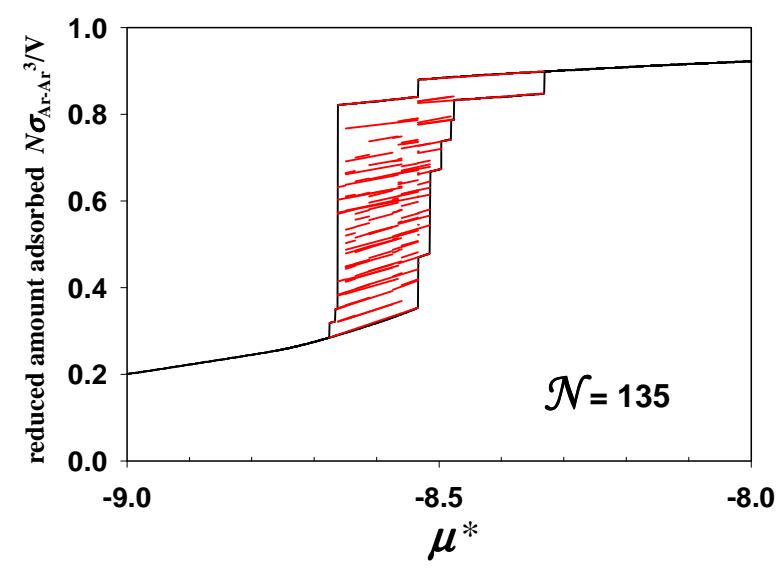

Figure 5: Monte Carlo simulation result of fluid adsorption in a heterogeneous pore comprising 15 domains, taking into account the interdependence between domains. Black continuous lines: main adsorption/desorption isotherms. Red portions of line: all (135) possible metastable states for this system.

These results are typical for a pore with a dozen domains, but details in the number and positions of the metastable states are dependent on the particular distribution of the heterogeneities in the pore. This effect is expected to be minimized for large pores containing a large number of heterogeneities, closer to experimental situations. As a consequence, an appropriate finite size analysis has been done to extrapolate to large systems. To do this analysis, and for each system size considered, an appropriate average over disorder (distribution of heterogeneities) has also to be performed to minimize spurious effects due to the choice of particular configurations for the heterogeneities. Previous works [72-76] have shown that the number of metastable states is expected to increase exponentially with system size (number of domains), and that the appropriate self-averaging quantity is the corresponding normalized logarithm. We thus introduce the quenched complexity as $\Sigma=\lim _{N \rightarrow \infty} \frac{1}{N} \overline{\ln \mathcal{N}}$ where $\mathcal{N}$ is the number of metastable states for each system, $N$ is the number of domains (measure of the system size), and the bar denotes the average over 
disorder. In order to get insight in the distribution of the metastable states within the hysteresis loop, the local quenched complexity is introduced:

$$
\sum^{\infty}(\rho ; \mu)=\lim _{N \rightarrow \infty} \frac{1}{N} \overline{\ln \mathcal{N}(\rho ; \mu ; N)}
$$

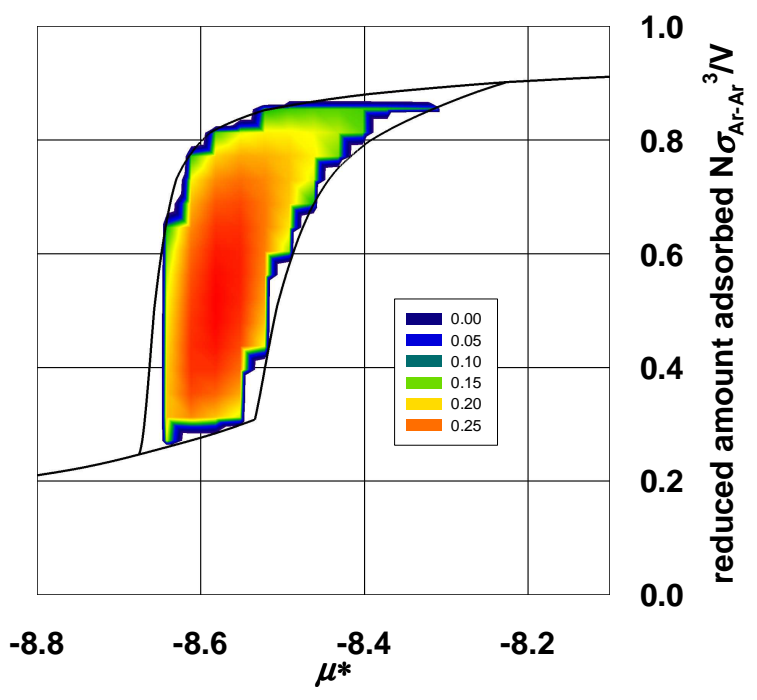

Figure 6: Local quenched complexity (see Eq 1) obtained by Monte Carlo simulation result of fluid adsorption in a heterogeneous pore comprising 15 domains, taking into account the interdependence between domains. The roughness of the periphery is due to the discretization used for numerical calculations. Solid lines: main adsorption/desorption isotherms.

Figure 6 shows the results for $N=25$. This system size allows a good compromise between large $N$ and a large number of disorder realizations $\left(5 \times 10^{4}\right)$ to increase statistical accuracy. As can be seen, the complexity presents a typical shape with maximal values in the hysteresis loop. An interesting feature is that the location where $\Sigma(\rho ; \mu)=0$ closely follows the gaslike and liquidlike adsorption/desorption branches, within statistical accuracy and discretization: this result is in agreement with what has been observed in other systems $[72,73,75]$. 


\section{CONCLUSION}

A molecular simulation approach has been used to model simple fluid adsorption in heterogeneous tubular pores mimicking mesoporous materials like MCM-41 or porous silicon (with typical argon/silica interaction parameters). A hysteresis loop is observed in adsorption / desorption cycles, which is closely connected to the appearance of many metastable states. The density of these metastable states is studied in the $\mu-\rho$ plane. Although this quantity is not directly accessible to experiments, it is of primary theoretical importance to understand the location of the hysteresis and the shape of the scanning curves as determined experimentally. As a conclusion, the main adsorption/desorption hysteresis loop appears as the envelope of the numerous metastable states appearing in the system.

This work focuses essentially on one-dimensional systems. From this point of view, the results are complementary of other fundamental studies focusing for instance on the effect of surface and finite size on the occurrence of true phase transitions.[77] This fundamental work can also, on the other hand, be considered for more applicative studies. As has been suggested, it could be applied to strongly adsorbing systems like carbon dioxide or methane in carbonaceous materials for industrial applications (storage, gas shale, etc.).

\section{ACKNOWLEDGMENTS}

Dr. E. Kierlik and Dr. G. Tarjus are gratefully acknowledged for helpful discussions. The simulations were performed thanks to grant from the Institut de Développement des Ressources en Informatique Scientifique IDRIS-CNRS, Orsay, France. 


\section{REFERENCES}

[1] Cushman J H. The Physics of Fluids in Hierarchical Porous Media: Angstroms to Miles. London: Kluwer Academic Publishers; 1997.

[2] Guéguen Y, Palciauskas V. Introduction to the physics of rock. Princeton: University Press; 1994.

[3] Gregg S J, Sing K S W. Adsorption, Surface Area and Porosimetry. New York: Academic Press; 1982.

[4] Rouquerol F, Rouquerol J, Sing K S W. Adsorption by Powders and Porous Solids. London: Academic Press; 1999.

[5] Legrand A P. The surface properties of silicas. Chichester: John Wiley \& Sons Ltd; 1998.

[6] Puibasset J, Pellenq R J-M. A comparison of water adsorption on ordered and disordered silica substrates. Phys. Chem. Chem. Phys. 2004;6:1933-1937.

[7] Pellenq R J-M, Roussel T, Puibasset J. Molecular simulations of water in hydrophobic microporous solids. Adsorption. 2008;14:733-742.

[8] Puibasset J, Pellenq R J-M. Grand Canonical Monte Carlo simulation study of water adsorption in silicalite at 300K. J. Phys. Chem. B. 2008;112:6390-6397.

[9] Puibasset J, Pellenq R J-M. Water confined in mesoporous silica glasses: Influence of temperature on adsorption/desorption hysteresis loop and fluid structure. Eur. Phys. J. Special Topics. 2007; 141:41-44.

[10] Bussai C, Vasenkov S, Liu H, Böhlmann W, Fritzsche S, Hannongbua S, Haberlandt R, Kärger J. On the diffusion of water in silicalite-1 : MD simulations using ab initio fitted potential and PFG NMR measurements. Appl. Catal. A: Gen. 2002;232:59-66. 
[11] Findenegg G H, Jähnert S, Akcakayiran D, Schreiber A. Freezing and melting of water confined in silica nanopores. Chem. Phys. Chem. 2008;9:2651-2659.

[12] Takei T, Musaka K, Kofuji M, Fuji M, Watanabe T, Chikazawa M, Kanazawa T. Changes in density and surface tension of water in silica pores. Colloid Polym. Sci. 2000;278:475-480.

[13] Trzpit M, Soulard M, Patarin J, Desbiens N, Cailliez F, Boutin A, Demachy I, Fuchs A H. The effect of local defects on water adsorption in silicalite-1 Zeolite: a joint experimental and molecular simulation study. Langmuir. 2007;23:10131-10139.

[14] Zhuravlev L T. The surface chemistry of amorphous silica. Zhuravlev model. Colloids Surf. A: Physicochem. Eng. Aspects. 2000;173:1-38.

[15] Morishige K, Nobuoka K. X-ray diffraction studies of freezing and melting of water confined in a mesoporous adsorbent (MCM-41). J. Chem. Phys. 1997;107:6965-6969.

[16] Bellissent-Funel M-C, Lal J, Bosio L. Structural study of water confined in porous glass by neutron scattering. J. Chem. Phys. 1993;98:4246-4252.

[17] Brovchenko I, Oleinikova A. Effect of pore size on the condensation/evaporation transition of confined water in equilibrium with saturated bulk water. J. Phys. Chem. B. 2011;115:9990-10000.

[18] Liu Y, Wilcox J. Effects of surface heterogeneity on the adsorption of CO2 in microporous carbons. Environ. Sci. Technol. 2013;46:1940-1947.

[19] Liu Y, Wilcox J. Molecular simulation of CO2 adsorption in micro- and mesoporous carbons with surface heterogeneity. Int. J. Coal Geol. 2012;104:83-95.

[20] Liu Y, Wilcox J. Molecular simulation studies of CO2 adsorption by carbon model compounds for carbon capture and sequestration applications. Environ. Sci. Technol. 2013;47:95-101. 
[21] Firouzi M, Wilcox J. Molecular simulation of carbon dioxide transport and storage in porous carbon-based materials. Micropor. Mesopor. Mater. 2012;158:195-203.

[22] Firouzi M, Wilcox J. Slippage and viscosity predictions in carbon micropores and their influence on CO2 and CH4 transport. J. Chem. Phys. 2013;138:064705.

[23] Kierlik E, Puibasset J, Tarjus G. Effect of the reservoir size on gas adsorption in inhomogeneous porous media. J. Phys.: Condens. Matter. 2009;21:155102.

[24] Puibasset J, Kierlik E, Tarjus G. Influence of reservoir size on the adsorption path in an ideal pore. J. Chem. Phys. 2009;131:124123.

[25] Nguyen V T, Do D D, Nicholson D. Monte Carlo simulation of the gas-phase volumetric adsorption system: effects of dosing volume size, incremental dosing amount, pore shape and size, and temperature. J. Phys. Chem. B. 2011;115:7862-7871.

[26] Coasne B, Gubbins K E, Pellenq R J-M. Domain theory for capillary condensation hysteresis. Phys. Rev. B. 2005;72:024304.

[27] Coasne B, Pellenq R J-M. Grand canonical Monte Carlo simulation of argon adsorption at the surface of silica nanopores: effect of pore size, pore morphology, and surface roughness. J. Chem. Phys. 2004;120:2913-2922.

[28] Coasne B, Galarneau A, Pellenq R J-M, Di Renzo F. Adsorption, intrusion and freezing in porous silica: the view from the nanoscale. Chem. Soc. Rev. 2013;42:4141.

[29] Allen M P, Tildesley D J. Computer Simulation of Liquids. Oxford: Clarendon Press; 1987.

[30] Frenkel D, Smit B. Understanding Molecular Simulation. London: Academic Press; 1996.

[31] Nicholson D, Parsonage N G. Computer Simulation and the Statistical Mechanics of Adsorption. London: Academic Press; 1982. 
[32] Bock H, Schoen M. Phase behavior of a simple fluid confined between chemically corrugated substrates. Phys. Rev. E. 1999;59:4122-4136.

[33] Kuchta B, Firlej L, Marzec M, Boulet P. Microscopic mechanism of adsorption in cylindrical nanopores with heterogeneous wall structure. Langmuir. 2008;24:4013-4019.

[34] Coasne B, Hung F R, Siperstein F R, Gubbins K E. Molecular simulation of gas adsorption in realistic models of silica nanopores. Ann. Chim. Sci. Mat. 2005;30:375-383.

[35] Coasne B, Hung F R, Pellenq R J-M, Siperstein F R, Gubbins K E. Adsorption of simple gases in MCM-41 materials: the role of surface roughness. Langmuir. 2006;22:194-202.

[36] Gelb L D, Gubbins K E, Radhakrishnan R, Sliwinska-Bartkowiak M. Phase separation in confined systems. Rep. Prog. Phys. 1999;62:1573-1659.

[37] Evans R, Marini Bettolo Marconi U, Tarazona P. Capillary condensation and adsorption in cylindrical and slit - like pores. J. Chem. Soc., Faraday Trans. 2. 1986;82:1763-1787.

[38] Morishige K, Shikimi M. Adsorption hysteresis and pore critical tempereture in a single cylindrical pore. J. Chem. Phys. 1998;108:7821-7824.

[39] Sonwane C G, Bhatia S K. Analysis of criticality and isotherm reversibility in regular mesoporous materials. Langmuir. 1999;15:5347-5354.

[40] Puibasset J. Pseudocritical or hysteresis temperature versus pore size for simple fluids confined in cylindrical nanopores. J. Chem. Phys. 2008;129:024705.

[41] Puibasset J. Influence of surface chemical heterogeneities on adsorption/desorption hysteresis and coexistence diagram of metastable states within cylindrical pores. J. Chem. Phys. 2006;125:074707.

[42] Kierlik E, Monson P A, Rosinberg M L, Tarjus G. Adsorption hysteresis and capillary condensation in disordered porous solids: a density functional study. J. Phys.: Condens. Matter. 2002;14:9295-9315. 
[43] Kierlik E, Monson P A, Rosinberg M L, Sarkisov L, Tarjus G. Capillary condensation in disordered porous materials : hysteresis versus equilibrium behavior. Phys. Rev. Lett. 2001;87:055701.

[44] Beck J S, Vartuli J C, Roth W J, Leonowicz M E, Kresge C T, Schmitt K D, Chu C T-W, Olson D H, Sheppard E W, McCullen S B, et al. A new family of mesoporous molecular sieves prepared with liquid cristal templates. J. Am. Chem. Soc. 1992;114:10834-10843.

[45] Kresge C T, Leonowicz M E, Roth W J, Vartuli J C, Beck J S. Nature. 1992;359:710712.

[46] Uhlir A. Electrolytic shaping of germanium and silicon. Bell Syst. Tech. J. 1956;35:333347.

[47] Coasne B, Grosman A, Ortega C, Simon M. Adsorption in noninterconnected pores open at one or at both ends: a reconsideration of the origin of the hysteresis phenomenon. Phys. Rev. Lett. 2002;88:256102.

[48] Grosman A, Ortega C. Nature of capillary condensation and evaporation processes in ordered porous materials. Langmuir. 2005;21:10515-10521.

[49] Grosman A, Ortega C. Capillary condensation in porous materials. Hysteresis and interaction mechanism without pore blocking/percolation process. Langmuir. 2008;24:39773986.

[50] Maddox M W, Olivier J P, Gubbins K E. Characterization of MCM-41 using molecular simulation: heterogeneity effects. Langmuir. 1997;13:1737-1745.

[51] Edler K J, Reynolds P A, White J W. Small-angle neutron scattering studies on the mesoporous molecular sieve MCM-41. J. Phys. Chem. B. 1998;102:3676-3683.

[52] Berenguer-Murcia A, Garcia-Martinez J, Cazorla-Amoros D, Martinez-Alonso A, Tascon J M D, Linares-Solano A. About the exclusive mesoporous character of MCM-41. In: 
F. Rodriguez Reinoso, B. McEnaney, J. Rouquerol and K. Unger, editors. Studies in Surface Science and Catalysis. Vol. 144. Amsterdam: Elsevier Science; 2002. p. 83-90.

[53] Fenelonov V B, Derevyankin A Y, Kirik S D, Solovyov L A, Shmakov A N, Bonardet JL, Gedeon A, Romannikov V N. Comparative textural study of highly ordered silicate and aluminosilicate mesoporous mesophase materials having different pore sizes. Micropor. Mesopor. Mater. 2001;44-45:33-40.

[54] Sonwane C G, Jones C W, Ludovice P J. A model for the structure of MCM-41 incorporating surface roughness. J. Phys. Chem. B. 2005;109:23395-23404.

[55] Puibasset J. Capillary condensation in a geometrically and a chemically heterogeneous pore: a molecular simulation study. J. Phys. Chem. B. 2005;109:4700-4706.

[56] Puibasset J. Phase coexistence in heterogeneous porous media : a new extension to Gibbs Ensemble monte carlo simulation method. J. Chem. Phys. 2005;122:134710.

[57] Puibasset J. Grand potential, Helmholtz free energy, and entropy calculation in heterogeneous cylindrical pores by the grand canonical Monte Carlo simulation method. J. Phys. Chem. B. 2005;109:480-487.

[58] Puibasset J. Thermodynamic characterization of fluids confined in heterogeneous pores by Monte Carlo simulations in the grand canonical and the isobaric-isothermal ensembles. J. Phys. Chem. B. 2005;109:8185-8194.

[59] Sethna J P, Dahmen K, Kartha S, Krumhansl J A, Roberts B W, Shore J D. Hysteresis and hierarchies : dynamics of disorder - driven first order phase transformations. Phys. Rev. Lett. 1993;70:3347-3350.

[60] Everett D H, Whitton W I. A general approach to hysteresis. Trans. Faraday Soc. $1952 ; 48: 749$.

[61] Everett D H, Smith F W. A general approach to hysteresis. Part2: Development of the domain theory. Trans. Faraday Soc. 1954;50:187. 
[62] Everett D H. A general approach to hysteresis. Part3: Formal treatment of the independent domain model of hysteresis. Trans. Faraday Soc. 1954;50:1077.

[63] Mason G. The effect of pore space connectivity on the hysteresis of capillary condensation in adsorption desorption isotherms. J. Colloid Interface Sci. 1982;88:36-46.

[64] Mason G. A model of adsorption - desorption hysteresis in which hysteresis is primarily developed by the interconnections in a network of pores. Proc. R. Soc. London A. $1983 ; 390: 47-72$.

[65] Swift M R, Cheng E, Cole M W, Banavar J R. Phase transitions in a model porous medium. Phys. Rev. B. 1993;48:3124.

[66] Kierlik E, Rosinberg M L, Tarjus G, Viot P. Equilibrium and out-of-equilibrium (hysteretic) behavior of fluids in disordered porous materials : theoretical predictions. Phys. Chem. Chem. Phys. 2001;3:1201-1206.

[67] Detcheverry F, Kierlik E, Rosinberg M L, Tarjus G. Local mean-field study of capillary condensation in silica aerogels. Phys. Rev. E. 2003;68:061504.

[68] Detcheverry F, Kierlik E, Rosinberg M L, Tarjus G. Gas adsorption and desorption in silica aerogels: a theoretical study of scattering properties. Phys. Rev. E. 2006;73:041511.

[69] Naumov S, Khokhlov A, Valiullin R, Kärger J, Monson P A. Understanding capillary condensation and hysteresis in porous silicon: Network effects within independent pores. Phys. Rev. E. 2008;78:060601.

[70] Puibasset J. Adsorption/desorption hysteresis of simple fluids confined in realistic heterogeneous silica mesopores of micrometric length: a new analysis exploiting a multiscale Monte Carlo approach. J. Chem. Phys. 2007;127:154701.

[71] Puibasset J. Monte Carlo multiscale simulation study of argon adsorption/desorption hsyteresis in mesoporous heterogeneous tubular pores like MCM-41 or oxidized Porous silicon. Langmuir. 2009;25:903-911. 
[72] Detcheverry F, Rosinberg M L, Tarjus G. Metastable states and T = 0 hysteresis in the random-field Ising model on random graphs. Eur. Phys. J. B. 2005;44:327-343.

[73] Pérez-Reche F J, Rosinberg M L, Tarjus G. Numerical approach to metastable states in the zero-temperature random-field Ising model. Phys. Rev. B. 2008;77:064422.

[74] Cherrier R, Dean D S, Lefèvre A. The number of metastable states in the generalized random orthogonal model. J. Phys. A: Math. Gen. 2003;36:3935-3945.

[75] Illa X, Rosinberg M-L, Vives E. Influence of the driving mechanism on the response of systems with athermal dynamics: the example of the random-field Ising model. Phys. Rev. B. 2006;74:224403.

[76] Puibasset J. Counting metastable states within the adsorption/desorption hysteresis loop: a molecular simulation study of confinement in heterogeneous pores. J. Chem. Phys. 2010;133:104701.

[77] Winkler A, Wilms D, Virnau P, Binder K. Capillary condensation in cylindrical pores: Monte Carlo study of the interplay of surface and finite size effects. J. Chem. Phys. 2010;133:164702. 